\title{
Scientific Automation Rises the Productivity of Production Facilities
}

\author{
U. Frank ${ }^{1}$, H. Anacker ${ }^{2}$, and Dirk Bielawny ${ }^{3}$ \\ ${ }^{1}$ Beckhoff Automation GmbH, Eiserstraße 5, 33415 Verl, Germany \\ u.frank@beckhoff.com \\ ${ }^{2}$ Heinz Nixdorf Institute Paderborn, Product Engineering, \\ University of Paderborn, Fuerstenallee 11, 33102 Paderborn \\ harald.anacker@hni.upb.de \\ ${ }^{3}$ Heinz Nixdorf Institute Paderborn, Control Engineering and Mechatronics, \\ University of Paderborn, Fuerstenallee 11, 33102 Paderborn \\ dirk.bielawny@hni.upb.de
}

\begin{abstract}
Manufacturer of production facilities and operating companies are subjected to increase competitive constraints. To be successful production facilities should work more productively and more environmentally friendly. Furthermore the quality of the produced goods should rise. To reach this manufacturer of production facilities ask for more efficient and intelligent automation technology. In the project "ScAut - Scientific Automation" the Beckhoff Automation $\mathrm{GmbH}$ - a producer and vendor of open automation systems based on PC Control technology - meets these requirements with Scientific Automation. Scientific Automation is the integration of automation software with findings from engineering science, which go beyond the limits of conventional control. The basis for this is the continually increasing performance of PCs. The power of the PC Control philosophy offers sufficient capacity to integrate numerous advanced functions beyond standard control. Scientific Automation complements the conventional areas of control technology such as PLC, Motion Control and control technology, for instance, with precise and fast measurement technology and the associated engineering algorithms.
\end{abstract}

Keywords: manufacturer, intelligent automation, scientific automation, control technologies, measurement technologies.

\section{Introduction}

Due to increasing competition and the approach of scarce resources, the manufacturers and operators of production facilities are attempting to develop more reliable, flexible, and more energy efficient manufacturing and assembly plants. The manufacturers and operators in automation technology see great potential in achieving these goals. They call for automation concepts and technologies that enable a self-contained anticipation of wear, in order to tie in maintenance procedures during pauses in the production line, to reduce emissions, to optimize energy consumption system wide and to avoid errors in production. 
The industry and scientific community agree that the progressive development of information technology and communication technology are the crucial point to meet these requirements. In the near future there will be fascinating perspectives for modern ma-chines and plant production that will go beyond current standards, for instance Intelligent technical systems with inherent partial intelligence. Keywords such as "Things that Think", "Cyber-Physical Systems" or "Industry 4.0" express this perspective. [1], [2], [3]. The development of such systems is the overall aim of the leading edge cluster it's OWL.

Information technology and also non-technical disciplines such as cognitive sciences, neurobiology and higher level mathematics bring forth a variety of methods, techniques, and procedures for developing technical systems that were previously only associated with biological systems. By integrating these properties, technical systems in the future will be able to react to altering environmental conditions, adjust their behaviour independently according to the situation, and to communicate, negotiate, cooperate with other technical systems in order to optimize its individual behaviour.

The intelligence of these systems is achieved through the constant integration of new functions. The realisation of these functions requires the following developments:

- new solutions for the electronic, software, and control technology as well as sensor technology especially for critical real-time applications;

- the integration of mathematical and scientific engineering methods, procedures, and algorithms as well as technology that is directly necessary for control;

- improved methods and procedures for data entry, data processing, data analysis, and data presentation (intelligent measuring technology and image processing systems;

- system-wide monitoring procedures.

As a provider of automation solutions, Beckhoff Automation is confronted with challenges to ease the applications of our solutions despite the increasing complexity of the challenges. The user provision that deals with new intelligent solutions and their integration is therefore critical (Figure1).

We confront these challenges in the ScAut Project, whose content and initial results are discussed in the framework of this paper.

In the innovation project "ScAut - Scientific Automation"1 the Beckhoff Automation $\mathrm{GmbH}$ - a producer and vendor of open automation systems based on PC Control technology - meets the defined requirements with Scientific Automation. Scientific Automation complements the conventional areas of control technology such as PLC's, Motion Control and control technology, for instance, with precise and fast measurement technology and the associated engineering algorithms. The ScAut Pro-

1 "This research and development project is funded by the German Federal Ministry of Education and Research (BMBF) within the Leading-Edge Cluster "Intelligent Technical Systems OstWestfalenLippe" (it's OWL) and managed by the Project Management Agency Karlsruhe (PTKA). The author is responsible for the contents of this publication." 
ject started in mid-2012 and is one of three core projects of the top cluster it's OWL (Intelligent Technical System OstWestfalenLippe). The top cluster it's OWL is the first major project in the framework of the future Industry 4.0.

A detailed explanation of the project and its objectives are presented in Chapter 2. In Chapter 3 we will present the PC-based control. Chapter 4 is the core of this paper. We will explain the scientific approaches as well as the exemplary use of pre-defined solution elements. To conclude, we will sum up the major points and give a short outlook on our future work.

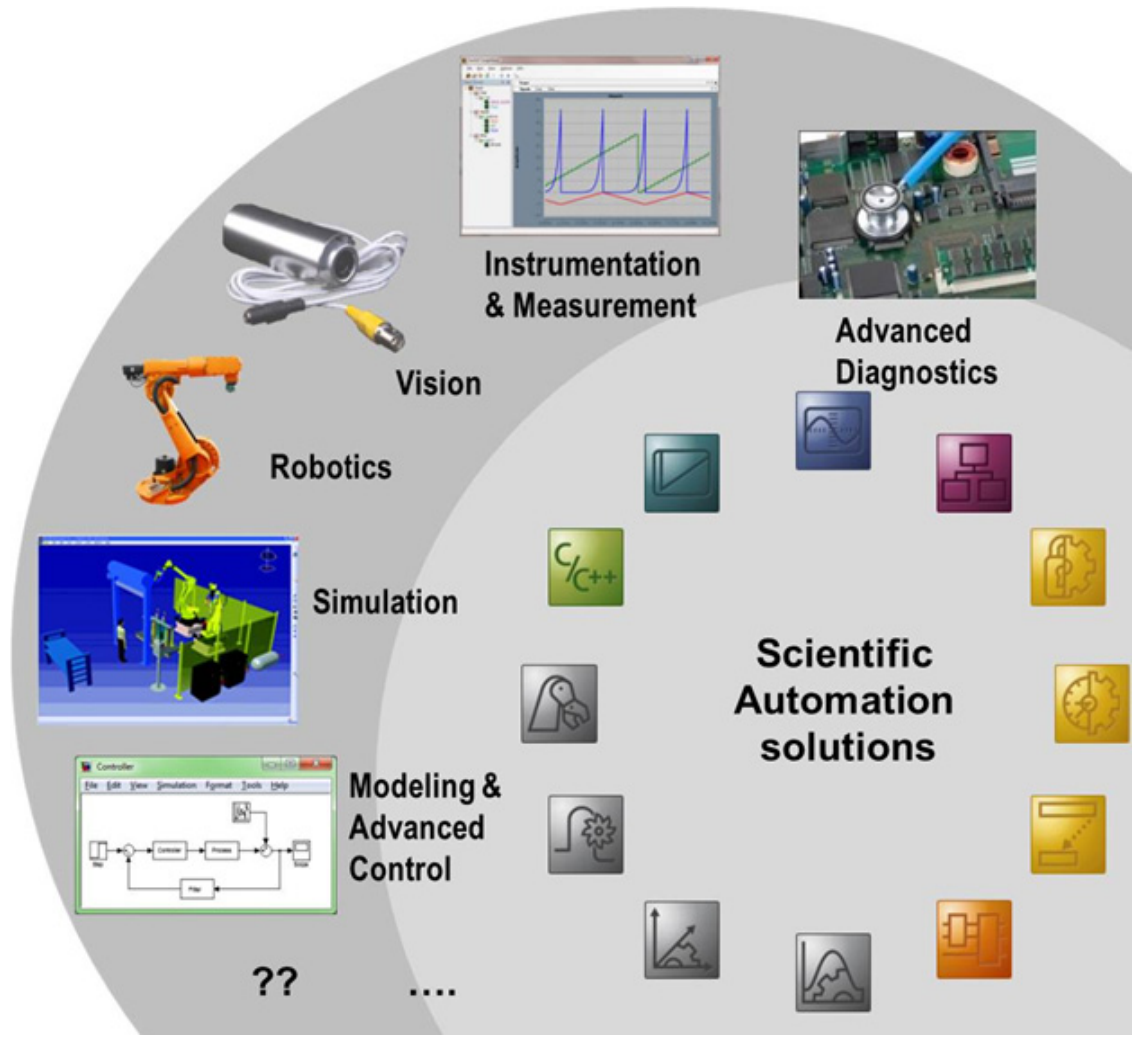

Fig. 1. Scientific Automation Platform

\section{Project Scientific Automation}

The aim of the project is to create a scientific automation platform for the development of real-time operating systems that have an inherent technical partial intelligence. The platform provides scientific automation solution elements in the form of hard-ware and software components, architectures of scientific automation systems, development tools, real-time run-time environment and a methodology. The platform is being developed by the interaction of three cooperative projects in cooperation with 
the cluster cross sectional project. The cross sectional cluster projects provide a successive comprehensive technology platform and broad base for personnel with knowledge and experience. They advise on application methods, processes and technologies and assist in the implementation of the platform. The overall objective is divided into the following sub-objectives:

\section{Target 1: Reference Architecture of Scientific Automation Systems}

Scientific Automation Systems are intelligent and adaptable. To realise these properties, the system must be built modular, be open and contain standardised interfaces. Scientific Automation Systems therefore contains modules that can be configured into a complete system. The systems have a hierarchical structure, which is composed of sub-modules. Each module implements one or more automation functions and has a specific behaviour and has defined standardised interfaces. The modules can be considered as solution elements. Scientific Automation Systems consist of preexisting solution elements (e.g. flow control, numerical control, power measurement terminal) and Scientific Automation Solution Elements. The current and potential relationships of the solution elements of a system define the system structure, the possible system structure (configurations) and the system behaviour.

The structure, the behaviour and the necessary interfaces of the solution elements are derived from the structure and the behaviour of the system. In addition, the integration mechanism is made available in order to share existing solution elements.

\section{Target 2: Construction Kit with Scientific Automation Solution Elements}

The objective is to define the engineering science, mathematical methods, procedures as well as technologies in technological roadmaps for the development and operation of the pilot applications, to specify the software components and hardware components necessary for their application and to provide them as Scientific Automation Solution Elements in the construction kit.

Technology-Roadmap: for the implementation of relevant methods in pilot applications, methods and procedures as well as technology, Technology-Roadmaps are collected and evaluated for functionality, performance, feasibility and beneficial factor for automation technology, potential re-usability and for market acceptance.

Scientific Automation solution elements: In the first step, Scientific Automation solution elements are prepared from the measurement fields and the condition monitoring. In the second step of the technology roadmap, the methods, processes and technologies are implemented with the highest ratings from the solution elements.

Construction kits: The Scientific Automation solution elements are to be made available to the developers of the construction kits. The kits can be realized through the database which the developer has access to. The search for the optimal solution element should be supported through the use of a semantic search.

\section{Target 3: Methods and Software Tools for the Design and Operation of Scientific Automation Systems}

Design tools, a runtime environment and methodology should be provided for the development and operation of Scientific Automation systems. 
Design tools: for the development of Scientific Automation systems, relevant design tools for modelling, simulation, analysis, optimization and implementation should be merged into a design environment. The solution elements are provided by the developers as construction kits. During the development, the developers use the construction kits depending on their design environment and in doing so develop efficient technical systems with inherent partial intelligence.

Run-time Environment: A real-time run-time environment is initialized for the operation of Scientific Automation systems. It is crucial that during operation of the system the real-time execution of the control program has access to the solution elements of the Scientific Automation construction kit as well as other solution elements of other construction kits and the appropriate software components of the solution elements.

Methodology: With the help of process models, methods and recommendations, third parties should also be able to develop Scientific Automation systems and thus enhance the competitiveness of their systems and their company.

The following pilot applications will demonstrate the power of the platform:

Intuitive, self-optimizing, energy efficient, profile processing machines (SCHIRMER Machines GmbH, Verl); Integrating intelligent measuring technology/software components in assembly and manufacturing plants (Hüttenhölscher Maschinenbau $\mathrm{GmbH}$ \& Co. KG, Verl); Woodworking machinery for the furniture industry (IMA Klessmann Holzbearbeitungssysteme, Lübbecke, Maschinenbau); Intelligent kitchens manufacturing with lot size 1 (Nobilia-Werke J. Stickling GmbH \& Co. KG, Verl).

\section{PC-Based Control}

In automation technology, the idea of converging information technology and automation technology with PC-based control was born in 1986 (see fig. 2).

As a pioneer of the industry standard IT hardware Beckhoff offers more than 25 years of PC-based control technology, which not only provides a huge increase in performance but has also exploited a medium for convergence of two worlds in the field of technology ever since. Already then it was recognized that the industrial PC facilitates high performance controls in a variety of form factors with an ideal IT orientation thanks to its openness. Presently and in the future, the increase in computing power will allow more and more tasks to be integrated in PC-based controls of a machine (figure 2). In addition to the classical sequence controller, steering control and traction control are already calculated directly by the PC. However in many cases, the available computing power is not used at all. There-fore the available computing power cannot exclusively be used for control but also for regulation, for measuring, and monitoring. 


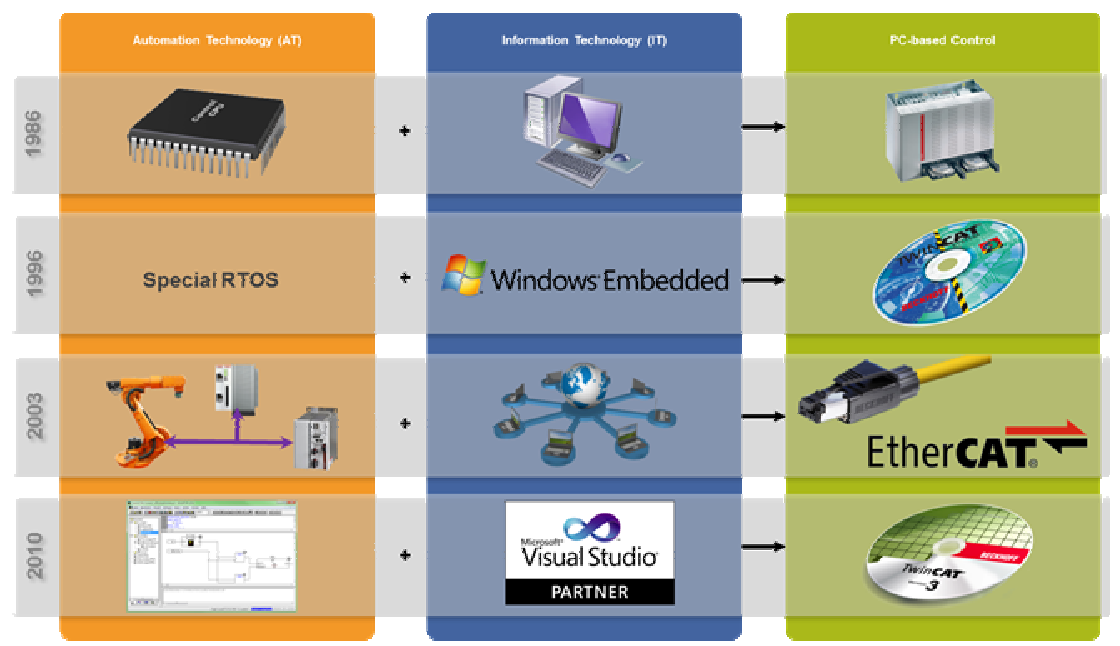

Fig. 2. PC-based Control uses the potential of the technology convergence of IT and AT for industrial applications

PC-based control systems consist of an Industrial PC, I/O modules (bus terminal), drive technology, and automation software. On the software side, the TwinCAT (The Windows Control and Automation Technology) automation suite is the heart of a Beckhoff PLC. The TwinCAT software systems turns virtually any PC-based system into a real-time control PC with many SPS, NC, CNC and other robotic run-time systems. TwinCAT serves as a run-time environment and also as a development platform.

On a PC, TwinCAT 3 can realise both the development as well as the simulation of technical systems. In enabling TwinCAT 3, a consistent model-based design from concept to implementation into an environment can be performed, which reduces costs and development times [2], [5], [6].

The PC-based platform, with its high performance processors and hardware platform, makes it principally possible to easily develop large projects, in which machines and control systems are simultaneously and reciprocally developed [7]. In practice, the steps in development look different up to now because the design of motion functions and control functions in the automation technology usually takes place independently from system design. The reason for this is that many industrial control systems and their software and hardware are largely incompatible with the development tools from other domains. A further complication is that currently the established development tools that do support aspects in the development of intelligent systems, such as modelling, simulation and optimization of flow controllers drive controllers, as well as the sup-port for methods, procedures and technologies for implementation such as self-optimization, multi-objective optimization, learning procedure or condition monitoring are not adequately considered in the area of design for automation systems. The project ScAut is to make a significant contribution to addressing this deficit. 


\section{Scientific Automation}

The core of Scientific Automation is the integration of different approaches of engineering sciences and intelligent measurements in automation software. The basic task of automation software is the controlling of system`s movements. The development of automation technologies of the last years - especially by the establishing of PCbased control systems - shows the integration of additional functions like contouring control or drive control in the sequence control based on the increasing available CPU resources.

The basis for the realization of such autonomous intelligent production systems are methods, procedures and algorithms of mathematical and scientific engineering. Beckhoff Automation will provide the so called solution elements by a combination of hardware modules with implemented adaptable software code. Definition of terms, examples for scientific approaches and examples of solution elements will explain in chapter 4.1. Content of chapter 4.2 is the exemplified use of the solution element ,balancing““.

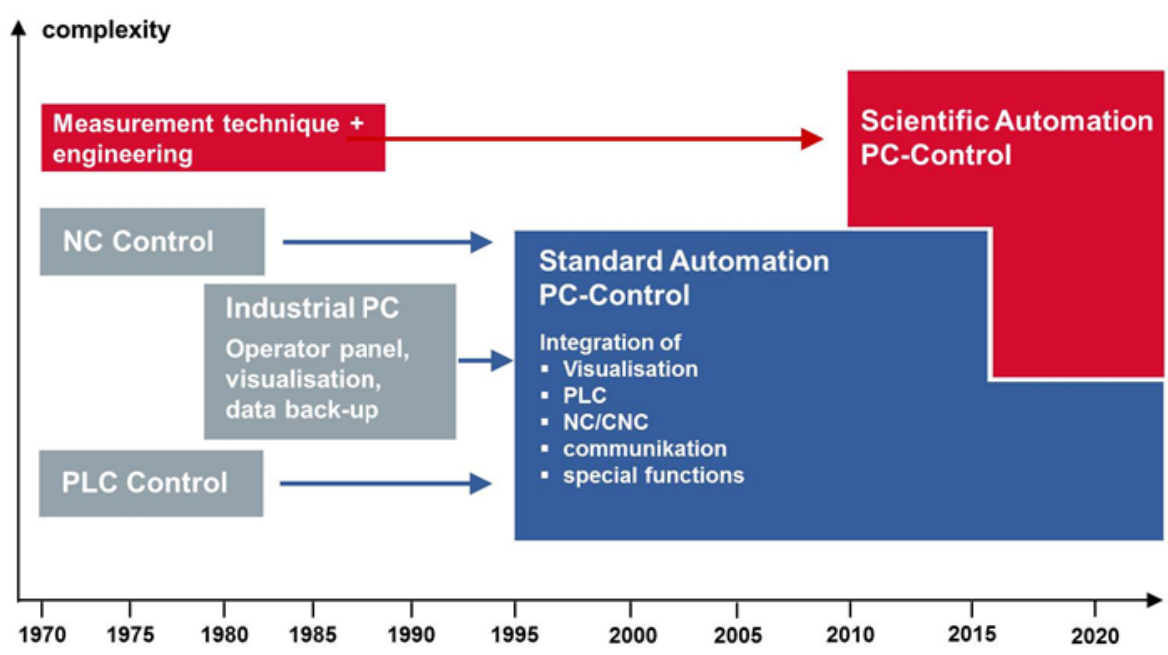

Fig. 3. From Standard Automation to Scientific Automation

\subsection{Scientific Approaches}

Scientific Automation provides new and enhanced functionalities for many application areas in the context of automation. Advanced methods, algorithms and technologies from various disciplines such as engineering, mathematics and computer science are explored and analyzed with regard to their possible use in Scientific Automation solution elements.

A method is "a particular procedure for accomplishing or approaching something, especially a systematic or established one" [9]. Here, we mainly - but not exclusively 
- consider mathematical or technical methods and procedures to solve certain problems or achieve certain goals. Besides, we regard mathematical and technical approaches (in contrast to particular procedures) as methods as well.

According to CORMEN ET.AL, we generally regard an algorithm as a welldefined procedure, i.e. a sequence of computational steps that uses a set of input values to produce a set of output values [10].

Technology is commonly defined as "machinery and equipment developed from scientific knowledge" [9]. In the context of ScAut, by technology we mean either specific machinery or scientific knowledge and methods closely related to certain machinery or equipment.

\subsubsection{Identification of Scientific Approaches}

The basis for identifying applicable methods for use in Scientific Automation solution elements is a comprehensive overview of the latest developments and research in disciplines that are related directly or indirectly to automation. "Scientific" approaches from various disciplines are to be exploited for their use in Automation.

ScAut will offer completely new features to automation systems. Thus a survey with the purpose of identifying methods, algorithms and technologies that are suitable for ScAut solution elements must not be limited to the classical areas of automation (e.g., control theory, signal processing and electrical drives). In order to realize intelligent and more efficient factories, new approaches for scientific automation solution elements are considered to be found in areas such as artificial intelligence, medical technology or economics. However, the focus is mainly on the areas of signal processing, cybernetics, computer vision, mathematics (e.g. optimization, stochastic), and data processing.

Starting with a specific application or a subject area, comprehensive inquiries are made based on expert interviews, literature and research reports. Thus, methods, algorithms and technologies are identified to create an overview of the state of the art regarding specific areas or applications. To find suitable approaches for ScAut solution elements, the identified methods and technologies are then analyzed and checked for their applicability.

\section{Classification, Evaluation and Selection}

So far about 500 methods, algorithms and technologies have been identified during the surveys for new approaches to ScAut solution elements. The identified items have been described, assigned to categories as well as potential application areas and merged to groups if possible. In a next step the methods are analyzed in order to obtain more detailed knowledge of their suitability for use in ScAut solution elements. There are some requirements towards ScAut solution elements allowing for decision whether an approach is suitable. Technical requirements such as real-time capability and computational complexity are of great importance. Usability and economic aspects have to be considered as well, among other things. Methods that meet these requirements can be further analyzed, described in detail and stored in a knowledge base. Thus, ScAut developers as well as users get quick access to information needed for their applications. 


\subsubsection{Promising Approaches and Examples}

Both the evaluation of the approaches already identified and the research for further methods are currently in progress. Nevertheless, there still are some promising approaches which show a great potential for the use in ScAut. In the following, we briefly present some examples. An more detailed example is given in chapter 4.2.

\section{Model-based Control}

Model-based approaches in both feedforward and feedback control offer interesting possibilities for applications in automation. Commonly established methods such as state regulators might serve as a useful basis for ScAut solution elements, which can be used in combination with more advanced approaches. Especially non-linear approaches, e.g., gain scheduling (see [11]) show great potential.

Another important concept is model predictive control. In model predictive control, optimal feedforward control signals are calculated on the basis of a model used to predict the future behavior of the system. An interesting example is given in [12]. The authors present an approach for model predictive control on the basis of differential dynamic programming. Possible applications in automation would be the efficient control of industrial robots.

A major drawback of the use of model-based approaches is the need for sufficiently accurate system models. ScAut can overcome this problem in different ways. Of course, users can create their own models using common tools, and import them to their Scientifc Automation applications.

But solution elements also could directly provide suitable (partial) models and support the user in selection and parameterization of the models. Other promising features are automatic generation and parameterization of plant models. An example for automatic model identification is the approach of Box-Jenkins [11]. Based on time series analysis, plant models as well as disturbance models can be generated automatically by this method through measuring of input and output signals. Although applying predefined input signals while measuring the outputs is preferred, the identification can take place even during normal operation of a plant.

\section{Examples in Context "Condition Monitoring“}

To ensure consistent production quality, a provision is used to monitor the conditions of machinery and the changes in the systems behaviour in order to introduce accompanying measures. Using sensors and supervisory equipment, the state of the machine and its components are collected and the data is analysed in information processing systems. The decisive factor for the reliability of the data analysed is the quality of the data collected. This depends entirely on the temporal accuracy of the data collection, the amount of data, the accuracy of the data and the amount of interference analysed.

The project ScAut found it necessary to filter out interferences and those with time stamps for collection, saving and analysis of data for solution elements such as bus terminals or data with high resolution and frequency. Secondly, insubstantial solution elements in the form of software are used. The Fast-Fourier-Transformation, the Hilbert envelope, or the digital Filter are for example employed. The algorithms are encapsulated in function blocks and are made available as solution elements in libraries for general use. 
A typical process for the realization of a digital filter is the Wattmeter method [13]. Both the amplitude and the phase of a sinusoidal signal can be deter-mined at a particular frequency at a mixed-voltage. The method is implemented as a function block named "Watt meter" and initialized as a solution element. This includes the state machine shown in Figure 5.

The evaluated values are assigned in the form of a pointer to an array of data; in addition, the number of available data is transferred into this array. The function block requires the values of the time stamp of a reference pulse detector and the next conversion cycle and the time of cycle in order to assign the time of the reference pulse and the function block.

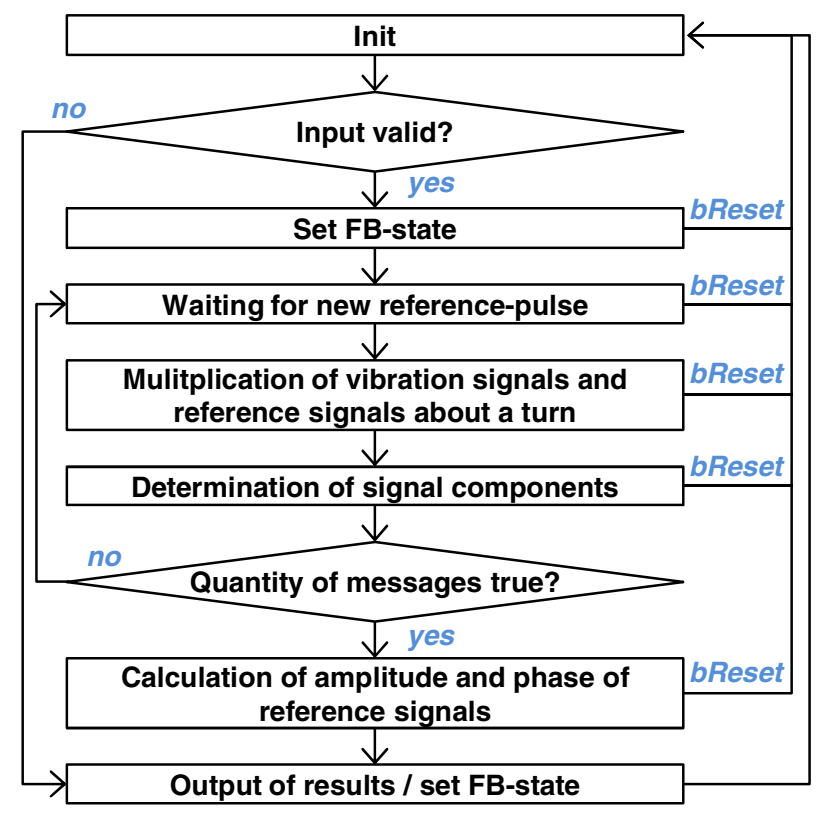

Fig. 4. Program sequence: Function block „Wattmeter“ [14]

\subsection{Application Example "Balancing"}

The use of ScAut solution elements are emphasized in balancing the use of spindles. The productivity, quality, durability, and cost-effectiveness of modern milling machines and work centres are rising steadily. Therefore motorized spindles with high revolutions are used. To achieve a high quality of machining operations, the spindles have to round and are not allowed to vibrate during the process.

Upon completion, all of the spindles will be tested, measured and balanced upon their dimensional accuracy, concentricity, balance, and durability. This takes place on a specially designed testing rig. The spindles are then put into operation and subjected to various tests. 
To control the testing rig, former stand-alone devices are used as well as dedicated systems that work in parallel with its own controls. In addition to its own configuration software and programming software, the system also has its own bus system in order to capture data from the connected sensors. These however lack flexibility due to the limited amount of analogue and digital input channels. Even the correlation of data from the "normal" control can only be realized with cross communication and heightened engineering effort.

The alternative to these "black box" systems is the integration of a condition monitoring system in the machine controls. Therefore the following two conditions have to be met:

1. The controller has to be a high performance controller in order to calculate the partially complex analysis algorithms and the control tasks.

2. The fieldbus system used has to allow the required data throughput and have a high determinism.

These requirements can be met with a PC controller and ScAut solution elements. EtherCAT meets the demands on the fieldbus system. EtherCAT has established itself as a fieldbus control and as a measurement fieldbus. Complex applications, like the integration of condition monitoring based on ScAut solution elements, are realizable with these ethernet-based high speed and deterministic fieldbus protocols. The functional principle of Ether-CAT makes possible payload rates of well over $90 \%$ and Fast Ethernet full-duplex and bus cycle times of a few microseconds. With EtherCAT oversampling technology, the buffering values directly in the EtherCAT slave are increased way beyond the actual bus cycles: Digital input bus terminals can deliver up to one million samples per second. Analogue signals may be sampled up to $100 \mathrm{kHz}$. Distributed clocks in EtherCAT slaves provide a network-wide time-synchronized data acquisition. The jitter is significantly below one microsecond, in most cases less than 100 nanoseconds. Moreover, the flexibility in the network topology and modularity of the bus terminals has to be mentioned. Using this process, each application is realized cost effectively.

The automation suite TwinCAT 3 (The Windows Control and Automation Technology) from the Beckhoff Automation realizes the real-time controls on an industrial or embedded PC, and also takes advantage of today's multi-core CPUs (Figure 2). TwinCAT can outsource different arithmetic operations on different cores of control. For example, one core can be used for machine control and another core can be dedicated to a condition monitoring application. Even the calculation of a single algorithm in a separate core is possible.

With the help of scientific automation, the balancing process that includes the necessary measurement equipment can be integrated directly into the controls of the spindle testing rig. The basis for this is the use of PC-based control technology that is composed of an industrial PC, the EtherCAT fieldbus, bus terminal and the Automation suite TwinCAT 3. The system data can be recorded very precisely with a time stamp and analysed with the aid of oversampling and distributed clocks technology.

The balancing of spindles is made in several steps. First the vibration sensor (encoder) and the spindle are aligned. These are then calibrated. In addition, several tests 
are performed with and without test weights. Then the balancing is performed. Any errors that occur are recorded and analyzed.

The calibration has to be done before testing new spindles. Otherwise, the settings might be taken from existing measuring routes. Figure 6 shows the structure of the main program for balancing (see. [14]).

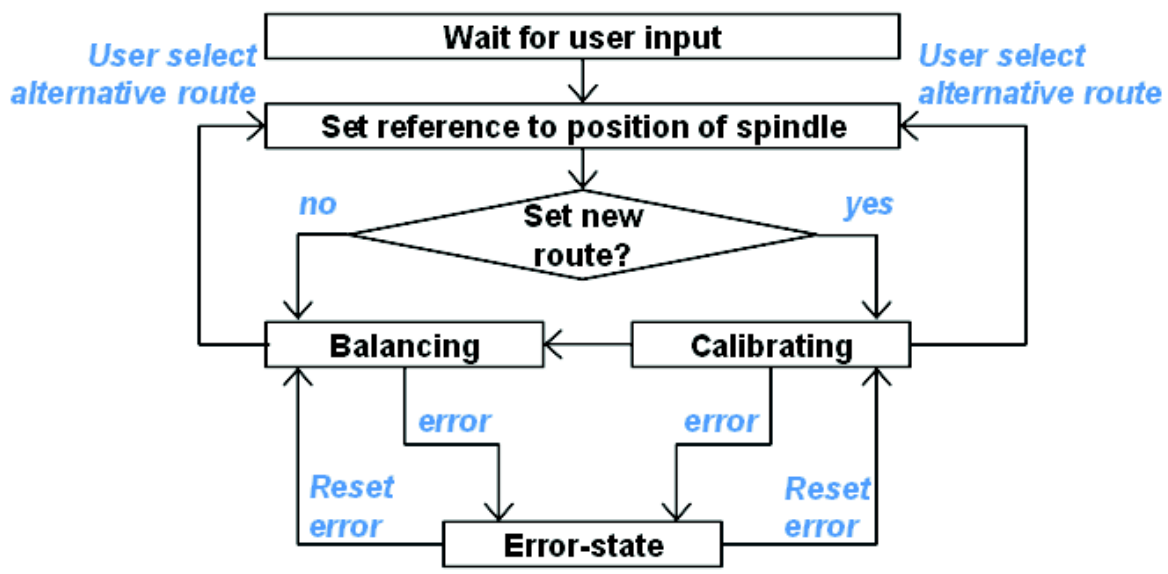

Fig. 5. Program sequence for the balancingoperation [14]

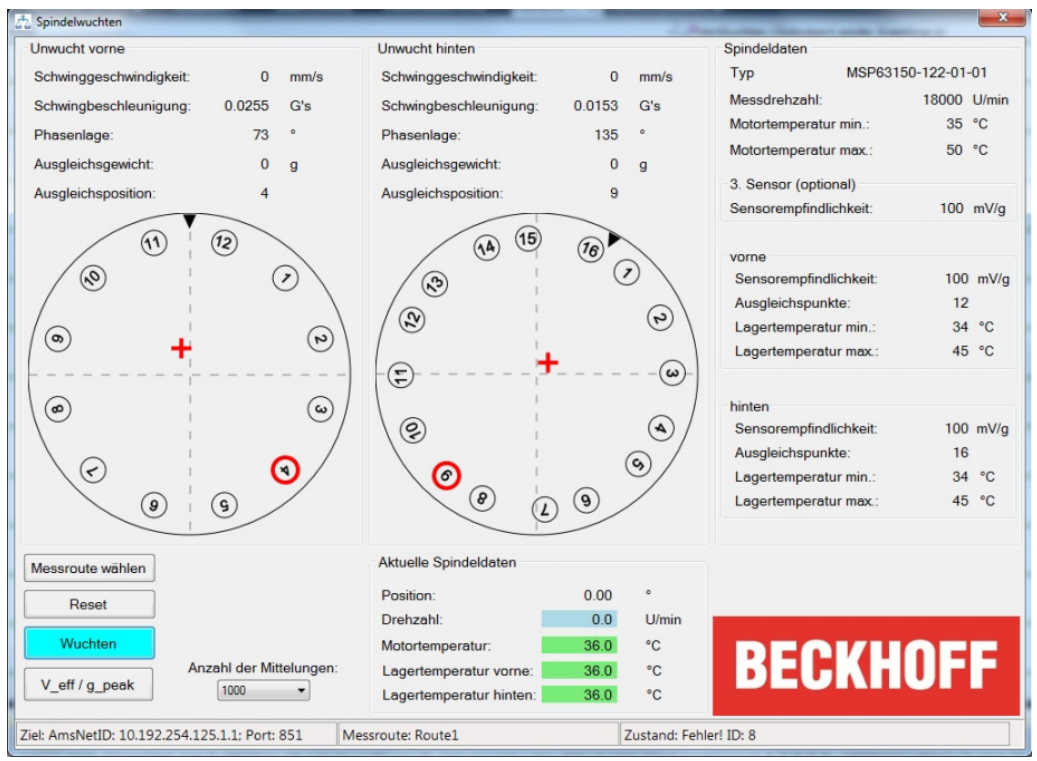

Fig. 6. User interface: Description of the results of the balancing operation [14]

In order to detect the imbalance, the vibrations of the spindle and the frequency of the vibrations have to be filtered out. This way the amplitude is the reference for the imbalance and the phase indicates the spatial position of the imbalance. This filtration 
is performed with the ScAut solution element "watt meter". The ScAut solution element can be integrated into the main program for balancing, which in turn can be interpreted as a ScAut solution element.

After performing the balancing process, the results are presented according to figure 6 . The phase of the imbalance, the necessary balance weight and the balancing position can be specified along with the information on the measured vibration velocity and the vibration acceleration.

The initial results show that the presented system can achieve a smaller number of measurement cycles, with a lower residual imbalance and a better overall result than the former solutions with stand-alone devices. Consequently, higher quality spindles at lower costs and fewer balancing process and simpler engineering can be reached through integrated solution.

\section{Conclusions}

The expansion of Automation Technology around Scientific Automation has many advantages. With the use of procedures and technologies such as self-optimization, learning, condition monitoring, analysis and reporting, production can be improved to react intelligently according to changing production conditions and to adapt its behaviour appropriately to its situation. This can lead to dramatic energy savings, reduced machinery wear, just like in the example of the balance; the balancing process can be executed more precisely, faster, and with less complex engineering. In the next four years, the project ScAut will do research in Scientific Automation solutions and make a Scientific Automation platform available to third parties.

\section{References}

1. Broy, M. (Hrsg.): Cyber-Physical Systems - Innovation durch softwareintensive eingebettete Systeme. acatech diskutiert. Springer, Berlin (2010)

2. Gausemeier, J., Rammig, F.J., Schäfer, W. (Hrsg.): Selbstoptimierende Systeme des Maschinenbaus. Definitionen, Anwendungen, Konzepte. HNI-Verlagsschriftenreihe, Band 234, Paderborn (2009)

3. Schmitt, R., et al.: Selbstoptimierende Produktionssysteme. In: Brecher, C. (Hrsg.): Integrative Produktionstechnik für Hochlohnländer. Springer, Berlin (2011)

4. Westkämper, E., Zahn, E. (Hrsg.): Wandlungsfähige Produktionsunternehmen - Das Stuttgarter Unternehmensmodell. Springer, Berlin (2009)

5. Vogel-Heuser, B., Braun, S., Kormann, B.: Implementation and evaluation of UML as modeling notation in object oriented software engineering for machine and plant automation. In: 18th World Congress of International Federation of Automation Control (IFAC), Milano (2011)

6. Papenfort, J.: Effektives Software-Engineering. In: Elektro Automation. Konradin Mediengruppe, Leinfelden Echterdingen, 11S (2012)

7. Papenfort, J.: Steuerung und Motion Control auf dem PC, geht das? In: Krammer, E. (Hrsg.): Automatisierungstechnik Aktuell-Ausgabe 2005-Trends, Produkte und Entscheidungshilfen, Oldenbourg-Industrie, München (2005) 
8. Haufe, J., Schneider, P., Donath, U., Reitz, S.: Simulationsgestütztes Prototyping und Optimierung von Maschinensteuerungen. In: Advances in Simulation and Logistics Applications. Fraunhofer IRB Verlag, Stuttgart (2008)

9. Stevenson, A. (ed.): Oxford Dictionary of English, 3rd edn. Oxford University Press (2010)

10. Cormen, T.H., Leiserson, C.E., Rivest, R.L., Stein, C.: Introduction to algorithms, 2nd edn. The MIT Press (2001)

11. Rugh, W., Shamma, J.: Research on gain scheduling. Automatica 36(10) (2000)

12. Julier, S., Uhlmann, J., Durrant-Whyte, H.: A New Approach for Filtering Nonlinear Systems, Seattle (1995)

13. Lingener, A.: Auswuchten - Theorie und Praxis, 1. Auflage. Springer, Heidelberg (2007)

14. Dresselhaus, P., Frank, U., Papenfort, J.: Scientific Automation erhöht Zuverlässigkeit Integration von Condition Monitoring in die Automatisierungstechnik. In: WT Werkstatttechnik Online Jahrgang 103. Springer VDI-Verlag, Düsseldorf (2013) 